\title{
Immunosuppressive Therapy in Myocarditis
}

\author{
Andrea Frustaci, MD; Cristina Chimenti, MD, PhD
}

\begin{abstract}
Although there is general agreement on the favorable effect of immunosuppression in eosinophilic, granulomatous, giant-cell myocarditis and in lymphocytic myocarditis associated with connective tissue disorders and with rejection of a transplanted heart, its therapeutic role in lymphocytic inflammatory cardiomyopathy (ICM) is still debated. Previous retrospective studies reported a relevant clinical benefit in $90 \%$ of patients with virus-negative ICM and no response or cardiac impairment in $85 \%$ of those with virus-positive ICM following immunosuppression. Other studies identified cardiomyocyte HLA upregulation as an additional indicator of ICM susceptibility to immunosuppressive therapy. Recently in a single-center randomized prospective double-blind trial using a combination of prednisone and azathioprine in addition to supportive treatment in 85 virus-negative ICM patients, a significant improvement in left ventricular (LV) ejection fraction and a significant reduction in LV dimensions in $88 \%$ of 43 treated patients compared with 42 patients receiving placebo who showed a cardiac impairment in $83 \%$ of cases (TIMIC study) was reported. These data confirm the efficacy of immunosuppression in virus-negative ICM. Lack of response in $12 \%$ of cases suggests the presence of unscreened viruses or mechanisms of damage and inflammation not susceptible to immunosuppression. Recovery of cardiac function in responders to immunosuppression was associated with inhibition of cardiomyocyte death, increased cell proliferation and with newly synthesized contractile material. (Circ J 2015; 79: 4-7)
\end{abstract}

Key Words: Heart failure; Immunosuppressive therapy; Myocarditis; Viruses

M yocarditis is an inflammatory disease of the heart caused by viral, bacterial and fungal infections, systemic diseases, autoimmune dysregulation, drugs and toxins. From the clinical point of view, it ranges from subclinical paucisymptomatic forms to life-threatening arrhythmias, ${ }^{1}$ cardiogenic shock and sudden death.

Although in approximately $40 \%$ of cases the acute myocarditis resolves spontaneously, ${ }^{2}$ in the remaining patients it evolves to a chronic phase as a consequence of an abnormal immune response, with ventricular dilation, reduced contractility, and clinical progression to heart failure..$^{3,4}$

Despite the advancement of diagnostic techniques in defining the etiology of myocarditis, a specific standardized treatment is not yet available. This is mainly related to the still unknown mechanisms regulating the normal or abnormal host immune response leading either to virus elimination and spontaneous resolution of the inflammatory process or to immunemediated damage persisting with or without viral clearance. In addition, the type of infectious agent and its prevalent mechanism of cell damage (ie, directly cytopathic or immune-mediated) could also affect the evolution of myocardial inflammation.

In particular, the use of immunosuppressive treatment for lymphocytic myocarditis is still controversial, ${ }^{5}$ both in children $^{6,7}$ and adults ${ }^{8,9}$ presenting with either cardiac arrhythmias ${ }^{10}$ or heart failure. ${ }^{11}$ Indeed, in the absence of specific markers of eligibility for this treatment, a large trial by Mason et al in the past produced misleading results showing the absence of evident improvement of survival in myocarditis patients treated with immunosuppressive drugs vs. placebo. ${ }^{9}$ For this reason, the use of immunosuppressive therapy is still confined to the treatment of eosinophilic, ${ }^{12}$ granulomatous ${ }^{13}$ and giant-cell myocarditis, ${ }^{14}$ as well as lymphocytic myocarditis associated with connective tissue diseases ${ }^{15}$ or with rejection of a transplanted heart. More recently Wojnicz et $\mathrm{al}^{16}$ in a randomized placebo-controlled study suggested that upregulation of HLA antigens in the myocardial tissue of patients with lymphocytic myocarditis may identify a homogeneous subgroup of inflammatory dilated cardiomyopathy sustained by an autoimmune mechanisms of damage, and may represent a marker of susceptibility to the treatment. However, in that study the presence of a viral genome in the myocardium was not investigated.

Our group, in both retrospective and prospective studies, identified the characteristics of patient responders to immunosuppressive therapy and the cellular and molecular mechanisms of cardiac recovery after immunosuppression.

Received October 30, 2014; accepted November 5, 2014; released online December 2, 2014

Cardiovascular, Respiratory, Nefrologic, Geriatric and Anestesiologic Sciences Department, La Sapienza University, Rome; IRCCS L.Spallanzani, Rome, Italy

Mailing address: Andrea Frustaci, MD, Cardiovascular Respiratory, Nefrologic, Geriatric and Anestesiologic Sciences Department, La Sapienza University, viale del Policlinico 155, 00161 Rome, Italy. E-mail: biocard@inmi.it

ISSN-1346-9843 doi:10.1253/circj.CJ-14-1192

All rights are reserved to the Japanese Circulation Society. For permissions, please e-mail: cj@j-circ.or.jp 

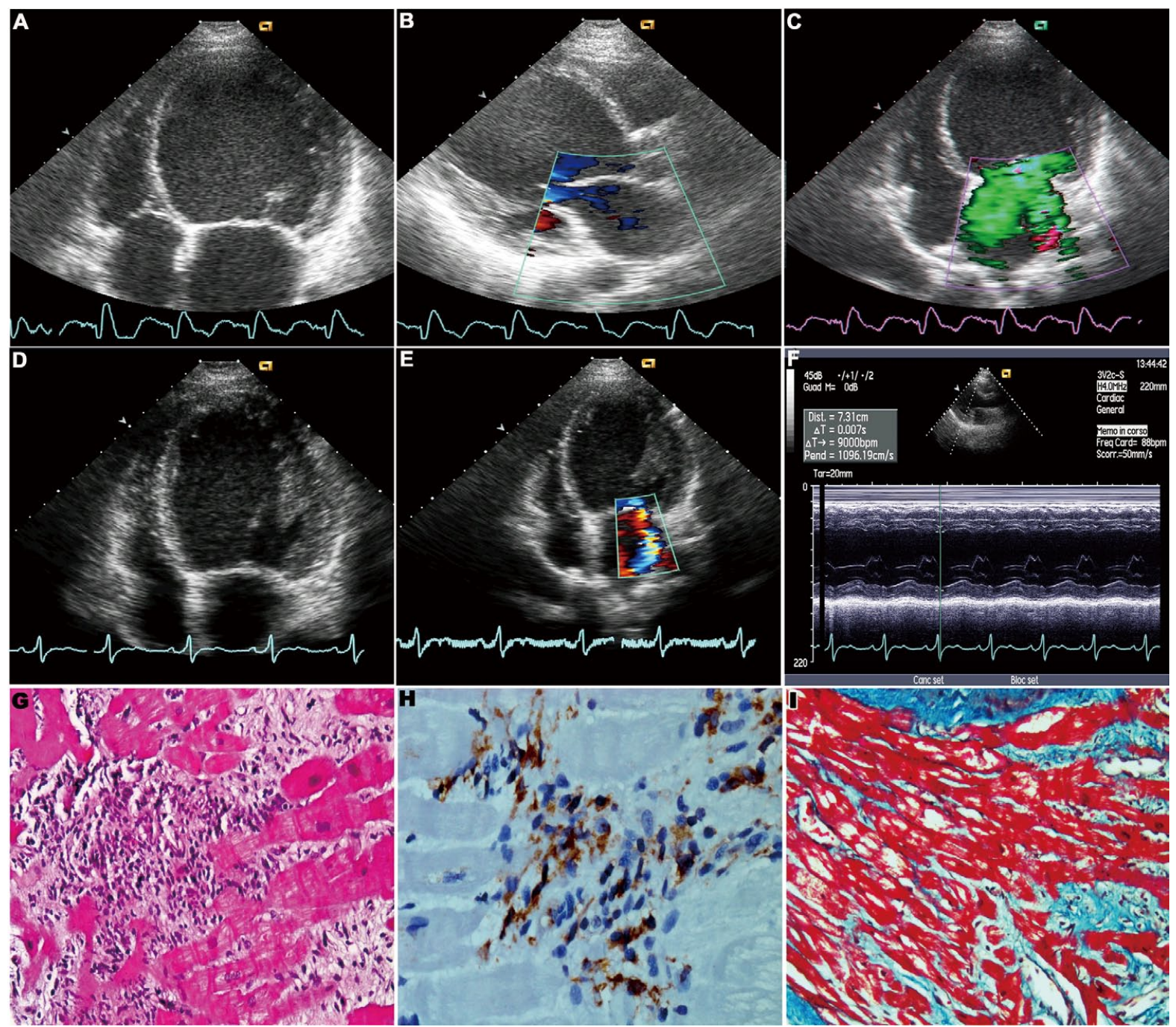

Figure. Echocardiographic and histologic evaluation of a 42-year-old man with chronic virus-negative inflammatory cardiomyopathy. Echocardiographic apical 4-chamber view (A) and long-axis view (B) showing severe LV dilation (EDD=90 mm) and dysfunction $(E F=18 \%)$, with remarkable mitral regurgitation (C, color Doppler imaging). These findings improved significantly after 6 months of immunosuppressive therapy, with EF increasing to $36 \%$ (D, apical 4-chamber view), mitral regurgitation reducing to mild (E, color Doppler imaging) and LVEDD reducing to $73 \mathrm{~mm}$ (F, M-mode imaging). Histology at baseline showed florid active lymphocytic myocarditis $(\mathbf{G}, \mathrm{H \& E}, \times 200)$ with overexpression of HLA-DR, denoting immune-mediated mechanism of damage $(\mathrm{H}$, immunoperoxidase, $\times 200$ ), progressing to healed myocarditis (I, Masson's trichrome, $\times 200$ ) after immunosuppression. Of note, the ECG recordings under the echocardiographic images show an intraventricular conduction disturbance (A-C) that disappeared after therapy (D-F). EDD, end-diastolic dimension; EF, ejection fraction: LV, left ventricular.

\section{Retrospective Study}

In our retrospective study, ${ }^{17}$ the virologic and immunologic profiles of patients with active lymphocytic myocarditis and chronic heart failure, responders and nonresponders to immunosuppressive therapy, were analyzed. The study group comprised 41 patients with a histological diagnosis of active myocarditis and characterized by progressive heart failure with an ejection fraction $(\mathrm{EF})<40 \%$, lasting $>6$ months despite conventional supportive therapy. All patients were similar in terms of the duration and severity of cardiac disease, histological findings and poor response to full supportive therapy. They received immunosuppressive therapy consisting of
$1 \mathrm{mg} \cdot \mathrm{kg}^{-1} \cdot$ day $^{-1}$ prednisone for 4 weeks followed by $0.33 \mathrm{mg}$. $\mathrm{kg}^{-1} \cdot \mathrm{day}^{-1}$ for $5 \mathrm{months}$ and $2 \mathrm{mg} \cdot \mathrm{kg}^{-1} \cdot \mathrm{day}^{-1}$ azathioprine for 6 months. Patients were classified as a responder if they had a decrease of at least 1 NYHA class and an improvement in EF $\geq 10 \%$ compared with baseline measures or a nonresponders if NYHA class and EF failed to improve or deteriorated or there were major events such as cardiogenic shock, heart transplantation or cardiac death. Among the 41 patients, 21 responded with a prompt improvement of EF and showed evidence of healed myocarditis in a control biopsy. Conversely, 20 patients failed to respond, and 12 of them remained unchanged, 3 underwent cardiac transplantation and 5 died, showing histological evolution towards dilated cardiomyopathy. Retro- 
spective PCR on frozen endomyocardial samples and evaluation of circulating cardiac autoantibodies on patients' sera showed that nonresponders had a high prevalence of viral genomes in the myocardium (85\%) and no detectable autoantibodies in the serum, whereas $90 \%$ of responders were positive for autoantibodies, with only $3(15 \%)$ presenting viral genomes on PCR analysis. Among the nonresponders, the myocardial persistence of enterovirus and adenovirus or their combination was associated with the worst clinical outcome. These data indicate that the absence of cardiac viral genomes is a prerequisite for the clinical use of immunosuppression while suggests the potential effect of antiviral agents for patients with virus-positive inflammatory cardiomyopathy (ICM). Interestingly, serology for cardiotropic viruses failed to predict the presence of viral genome in the myocardium. This result, also confirmed in a recent study, ${ }^{18}$ suggests that this tool cannot be used as an alternative to endomyocardial tissue PCR to diagnose viral myocarditis.

\section{Prospective Study}

To confirm our results in a prospective manner, we performed a randomized, double-blind, placebo-controlled single-center trial enrolling patients with myocarditis and chronic heart failure and submitting all patients with no evidence at PCR of a myocardial viral infection to immunosuppressive treatment. ${ }^{19}$ The 85 patients were treated prednisone $1 \mathrm{mg} \cdot \mathrm{kg}^{-1} \cdot \mathrm{day}^{-1}$ for 4 weeks followed by $0.33 \mathrm{mg} \cdot \mathrm{kg}^{-1} \cdot$ day $^{-1}$ for 5 months and azathioprine $2 \mathrm{mg} \cdot \mathrm{kg}^{-1} \cdot \mathrm{day}^{-1}$ for 6 months (43 patients, Group 1) or placebo (42 patients, Group 2) in addition to conventional therapy for heart failure. Primary outcome was the 6-month improvement in LV function. Group 1 showed a significant improvement in LVEF and a significant decrease in LV dimensions and volumes compared with baseline (Figure). Specifically, 38 of 43 patients on immunosuppressive therapy $(88 \%)$ showed an improvement in cardiac function and dimensions. The remaining 5 patients maintained a stable clinical picture and cardiac function parameters. Remarkably, even patients with severe, advanced disease (LVEDD $\leq 90 \mathrm{~mm}$ and LVEF $<20 \%$, Figure) significantly improved, being able to resume their previous work. The duration of heart failure did not correlate with the extent of recovery. None of the Group 2 patients at 6-month follow-up showed improvement in LVEF, which was significantly worsened compared with baseline. In particular, 35 of the Group 2 patient $(83 \%)$ showed further impairment of cardiac function while the remaining 7 patients remained unchanged. No major adverse reactions as a result of immunosuppression were registered. Histological analysis showed an active myocarditis with diffuse inflammatory infiltrates associated with focal necrosis of the adjacent myocytes (meeting the Dallas criteria) with interstitial and focal replacement fibrosis in most of the left and right ventricular specimens from all patients (Figure). The infiltrates included mainly activated $\mathrm{T}$ cells $(\mathrm{CD} 45 \mathrm{RO}+, \mathrm{CD} 3+)$ with a moderate number of cytotoxic lymphocytes (CD8+) and macrophages (CD68+).

Morphometric analysis showed no differences in terms of the extent of fibrosis and the number of inflammatory cells between Group 1 and Group 2 patients. Control histology at 1 and 6 months showed, in the 38 Group 1 patients who improved with immunosuppression, a healed myocarditis with disappearance of inflammatory infiltrates associated with interstitial and focal replacement fibrosis (Figure). In the 5 Group 1 patients who did not improve, myocardial inflammation had reduced or disappeared in the control biopsies but some de- generative changes of myocardiocytes were observed. In Group 2 patients, control biopsies were not dissimilar from baseline, showing persistence of myocarditis as well as expansion of interstitial and replacement fibrosis.

The results of this trial confirmed the positive effect of immunosuppression on recovery of LV function in a high percentage $(88 \%)$ of patients with virus-negative ICM. Remarkably, a striking improvement occurred even in patients with extreme LV dilatation and dysfunction. In this group of patients, myocardial inflammation was most likely the result of an immune-mediated injury towards segregated (ie, myosin) or new antigens shared with viral components (ie, antigenic mimicry).

The efficacy of immunosuppressive therapy in patients with myocarditis was confirmed in a recent meta-analysis ${ }^{20}$ of randomized controlled trials on immunosuppressive treatment for myocarditis in a selected 9 reports from 1966 to 2013 in which 342 patients were in the immunosuppressive treatment group and 267 patients in conventional treatment group. The immunosuppressive treatment group showed a significant improvement in LVEF at both short-term ( $\leq 3$ months) and long-term follow-up.

Finally, the recent position statement of the European Society of Cardiology ${ }^{21}$ and the JSC Guidelines ${ }^{22}$ both recommend the use of immunosuppression only after ruling out active infection on endomyocardial biopsy by PCR, as well as in proven autoimmune forms of myocarditis.

In our study, the lack of response in $12 \%$ of cases suggests the presence of unscreened viruses or mechanisms of damage and inflammation not susceptible to immunosuppression. With regard to undetected viral genomes, metagenomic assessment of the myocardial virome, including DNA and RNA extraction from PCR-negative endomyocardial biopsies and use of the GS-FLX platform, may identify new infectious agents and provide indications.

\section{Cellular Mechanisms of Cardiac Recovery}

The cellular mechanisms of cardiac recovery in patients with ICM treated with immunosuppression were analyzed, ${ }^{23}$ including cell death, activation of cell proliferation and reconstitution of cell myofibrillar content to clarify the effect of cell repair vs. cell proliferation or the possible contribution of cell death inhibition. The 10 responders, all showing the presence of circulating cardiac autoantibodies and absence of viral genomes in the myocardium at PCR analysis, and 10 nonresponders, characterized by worsening of LV dysfunction, absence of circulating cardiac autoantibodies, and by the presence of myocardial viral genomes ${ }^{13}$ were retrospectively studied in order to analyze the cellular events associated with the opposite clinical outcome. In all patients before treatment, transmission electron microscopy studies showed large cytoplasmic areas apparently empty or filled with fine granular material as a result of reduced myofibrillar content (myofibrillolysis). After 6-month immunosuppressive treatment, responders showed recovery of myofibrillar mass and architecture, whereas in the myocytes of nonresponders there was a further reduction of myofibrillar content. Further evidence of a strong activation of contractile protein synthesis comes, in responding patients, from molecular biology studies of the $\alpha$ - and $\beta$-isoforms of myosin heavy chain (MHC). The increased expression of $\alpha$-MHC and inhibition of $\beta$-MHC synthesis, with an enhanced $\alpha / \beta$ MHC ratio after effective treatment, strongly suggests gene activation of fetal protein isoforms that typically become operative in the process of cell repair. Both apoptotic and ne- 
crotic cell death of myocardiocytes were greater in baseline biopsies of responders and nonresponders than in controls, showing that myocyte loss is an important mechanism of myocardial damage in myocarditis with cardiac dysfunction. Importantly, after 6 months of effective immunosuppressive therapy, apoptosis and necrosis decreased by $85 \%$ and $62 \%$, respectively, but further increased by $42 \%$ and by $46 \%$, respectively, in follow-up biopsies of nonresponders. The number of cycling myocytes in baseline myocardial tissue of both responders and nonresponders was greater that in controls and significantly increased after immunosuppression in both groups, suggesting that in chronic myocarditis, as in other forms of heart failure, there is activation of myocyte regeneration in an attempt to compensate for cell loss. Thus, our study suggests that recovery of cardiac function in patients with myocarditis responding to immunosuppression is associated with remarkable cellular events, including strong inhibition of cell degeneration and death, activation of cell proliferation and mostly newly synthesized contractile elements.

\section{Conclusions}

Immunosuppressive therapy is an important resource in the management of chronic virus-negative ICM. Lack of identification of new or unconventional viral agents remains a major limit of this therapeutic approach, explaining the minor cohort of nonresponders. Future objective will be the development of molecular programs (ie, metagenomic assessment of the myocardial virome) able to detect elusive genome sequences.

\section{Source of Funding}

The study has been supported by the Grant RF-2009-1511346 and by the Grant RBFR081CCS from the Italian Ministry of Health.

\section{References}

1. Park H, Park H, Lee D, Oh S, Lim J, Hwang HJ, et al. Increased phosphorylation of $\mathrm{Ca}^{2+}$ handling proteins as a proarrhythmic mechanism in myocarditis. Circ J 2014; 78: 2292-2301.

2. Dec GW Jr, Palacios IF, Fallon JT, Aretz HT, Mills J, Lee DC, et al. Active myocarditis in the spectrum of acute dilated cardiomyopathies: Clinical features, histologic correlates, and clinical outcome. N Engl J Med 1985; 312: 885-890.

3. Miyagawa M, Yokoyama R, Nishiyama Y, Ogimoto A, Higaki J, Mochizuki T. Positron emission tomography-computed tomography for imaging of inflammatory cardiovascular diseases. Circ J 2014; 78: $1302-1310$.

4. Kawai S, Shimada T. Inflammation in takotsubo cardiomyopathy? Inquiry from "Guidelines for diagnosis and treatment of myocarditis (JCS 2009)". J Cardiol 2014; 63: 247-249.

5. Nakamura H, Kunitsugu I, Fukuda K, Matsuzaki M, Sano M. Diverse stage-dependent effects of glucocorticoids in a murine model of viral myocarditis. J Cardiol 2013; 61: 237-242.

6. Matitiau A, Perez-Atayde A, Sanders SP, Sluysmans T, Parness IA, Spevak PJ, et al. Infantile dilated cardiomyopathy: Relation of outcome to left ventricular mechanics, hemodynamics, and histology at the time of presentation. Circulation 1994; 90: 1310-1318.
7. Lee KJ, McCrindle BW, Bohn DJ, Wilson GJ, Taylor GP, Freedom RM, et al. Clinical outcomes of acute myocarditis in childhood. Heart 1999; 82: 226-233.

8. Parrillo JE, Cunnion RE, Epstein SE, Parker MM, Suffredini AF, Brenner M, et al. A prospective, randomized, controlled trial of prednisone for dilated cardiomyopathy. N Engl J Med 1989; 321: $1061-1068$.

9. Mason JW, O'Connell JB, Herskowitz A, Rose NR, McManus BM, Billingham ME, et al. A clinical trial of immunosuppressive therapy for myocarditis: The Myocarditis Treatment Trial Investigators. $N$ Engl J Med 1995; 333: 269-275.

10. Chimenti C, Calabrese F, Thiene G, Pieroni M, Maseri A, Frustaci A. Inflammatory left ventricular microaneurysms as a cause of apparently idiopathic ventricular tachyarrhythmias. Circulation 2001; 104: $168-173$.

11. Feldman AM, McNamara D. Myocarditis. N Engl J Med 2000; 343: $1388-1398$.

12. Frustaci A, Gentiloni N, Chimenti C, Natale L, Gasbarrini G, Maseri A. Necrotizing myocardial vasculitis in Churg-Strauss syndrome: Clinicohistologic evaluation of steroids and immunosuppressive therapy. Chest 1998; 114: 1484-1489.

13. Badorff C, Schwimmbeck PL, Kühl U, Gerhold M, Stein H, Schultheiss HP. Cardiac sarcoidosis: Diagnostic validation by endomyocardial biopsy and therapy with corticosteroids. Z Kardiol 1997; 86: 9-14 (in German).

14. Cooper LT Jr, Berry GJ, Shabetai R. Idiopathic giant-cell myocarditis: Natural history and treatment: Multicenter Giant Cell Myocarditis Study Group Investigators. N Engl J Med 1997; 336: 1860-1866.

15. Frustaci A, Gentiloni N, Caldarulo M. Acute myocarditis and left ventricular aneurysm as presentations of systemic lupus erythematosus. Chest 1996; 109: 282-284.

16. Wojnicz R, Nowalany-Kozielska E, Wojciechowska C, Glanowska G, Wilczewski P, Niklewski T, et al. Randomized, placebo-controlled study for immunosuppressive treatment of inflammatory dilated cardiomyopathy: Two-year follow-up results. Circulation 2001; 104: $39-45$.

17. Frustaci A, Chimenti C, Calabrese F, Pieroni M, Thiene G, Maseri A. Immunosuppressive therapy for active lymphocytic myocarditis: Virological and immunologic profile of responders versus nonresponders. Circulation 2003; 107: 857-863.

18. Mahfoud F, Gartner B, Kindermann M, Ukena C, Gadomski K, Klinkel K. Virus serology in patients with suspected myocarditis: Utility or futility? Eur Heart J 2011; 32: 897-903.

19. Frustaci A, Russo MA, Chimenti C. Randomized study on the efficacy of immunosuppressive therapy in patients with virus-negative inflammatory cardiomyopathy: The TIMIC study. Eur Heart J 2009; 30: 1995-2002.

20. Lu C, Qin F, Yan Y, Liu T, Li J, Chen H. Immunosuppressive treatment for myocarditis: A meta-analysis of randomized controlled trials. J Cardiovasc Med (Hagerstown) 2014 July 4, doi:10.2459/ JCM.0000000000000134.

21. Caforio AL, Pankuweit S, Arbustini E, Basso C, Gimeno-Blanes J, Felix SB, et al; European Society of Cardiology Working Group on Myocardial and Pericardial Diseases. Current state of knowledge on aetiology, diagnosis, management, and therapy of myocarditis: A position statement of the European Society of Cardiology Working Group on Myocardial and Pericardial Diseases. Eur Heart J 2013; 34: $2636-2648,2648 \mathrm{a}-2648 \mathrm{~d}$.

22. JCS Joint Working Group. Guidelines for diagnosis and treatment of myocarditis (JCS 2009): Digest version. Circ J 2011; 75: 734-743.

23. Frustaci A, Chimenti C, Pieroni M, Salvatori L, Morgante E, Sale P, et al. Cell death, proliferation and repair in human myocarditis responding to immunosuppressive therapy. Mod Pathol 2006; 19: $755-765$. 